\title{
Fractions continues normales dans un corps de fonctions hyperelliptiques
}

\author{
par \\ Isabelle Hardy, Yves Hellegouarch \\ et Roger Paysant-Le Roux (Caen)
}

Introduction. Soit $x$ une indéterminée. De nombreux auteurs [1]-[4], [9] ont étudié des séries de Laurent, $\alpha(x)=\sum_{h \geq-h_{0}} \gamma_{h} / x^{h}$, à coefficients dans un corps $k$, dont le développement en fraction continue est normal. La notion de normalité d'une série de Laurent est due à D. E. Knuth [6] qui observe que le premier quotient complet de $\alpha(x)$ est de degré 1 si et seulement si $\gamma_{1} \neq 0$, le deuxième quotient complet est de degré 1 si et seulement si $\gamma_{2}^{2}-\gamma_{1} \gamma_{3} \neq 0$, récursivement le nème quotient complet est de degré 1 si et seulement si un déterminant fonction de $\gamma_{1}, \ldots, \gamma_{n+1}$ est différent de zéro. On se propose dans une première partie de faire une étude un peu générale de la normalité dans le cadre des fonctions hyperelliptiques en caractéristique différente de 2 . Le théorème de Mason, tel qu'il est énoncé dans [5], permet de considérer le cas de la caractéristique infinie comme la limite lorsque $p$ tend vers l'infini du cas de la caractéristique finie. Grâce à ce théorème dans sa version en caractéristique infinie [7] et sa version en caractéristique finie [5], on montre qu'en caractéristique infinie et pour une large classe de fonctions, les degrés des quotients complets de ces fonctions sont majorés par une constante beaucoup plus petite que celle prévue par la théorie habituelle (cf. proposition 1) et que, en caractéristique finie, et pour ces mêmes types de fonctions, soit le résultat précédent tient, soit une certaine "équation de Pell" admet une solution non triviale (cf. théorème 1). Une conséquence de ce théorème est la suivante : si l'on prend $k=\mathbb{Q}$, si on se donne une fonction quadratique $\alpha$ sur $\mathbb{Q}(x)$, si l'on considère sa réduction modulo $p$, notée $\bar{\alpha}$ (ce que l'on peut faire pour $p$ assez grand), si on s'intéresse à la longueur de sa quasi-période $\pi_{p}(\bar{\alpha})$, alors, sous certaines hypothèses, on montre que $\pi_{p}(\bar{\alpha})$ tend vers l'infini avec $p$.

2000 Mathematics Subject Classification: Primary 30B70, 11A55, 11J70; Secondary 11Y65, 30Bxx. 
1. Développement en fraction continue. Soit $k$ un corps de caractéristique différente de deux. On note $\widetilde{K}=k((1 / x))$ le corps des séries de Laurent formelles en $1 / x$. Soit $\alpha \in \widetilde{K} \backslash\{0\}$. On note $E(\alpha)$ la partie polynomiale de $\alpha$ et on définit le degré de $\alpha \operatorname{par} \operatorname{deg} \alpha=h_{0}$ si $\alpha=$ $\sum_{h \geq-h_{0}} \gamma_{h} / x^{h}, \gamma_{h} \in k, \gamma_{-h_{0}} \neq 0$. On suppose maintenant que $\alpha \in \widetilde{K} \backslash k(x)$ et on pose $\alpha_{0}=\alpha$; on définit alors $\alpha_{1}$ par l'égalité

$$
\alpha=E(\alpha)+1 / \alpha_{1},
$$

et donc $\operatorname{deg} \alpha_{1}>0$; de même on définit $\alpha_{2}$ par l'égalité

$$
\alpha_{1}=E\left(\alpha_{1}\right)+1 / \alpha_{2}
$$

de façon générale, on définit $\alpha_{n+1}$ à partir de $\alpha_{n}$ par l'égalité

$$
\alpha_{n}=E\left(\alpha_{n}\right)+1 / \alpha_{n+1},
$$

ceci est possible car $\alpha \notin k(x)$.

On appelle développement en fraction continue de $\alpha$ la suite des polynômes $\left(E\left(\alpha_{n}\right)\right)_{n \geq 0}$ et on le note $\left[E\left(\alpha_{0}\right), E\left(\alpha_{1}\right), \ldots\right]$; l'élément $\alpha_{n}$ (resp. $\left.E\left(\alpha_{n}\right)\right)$ s'appelle le nème quotient complet (resp. incomplet) du développement en fraction continue de $\alpha$.

On appelle nème réduite du développement de $\alpha$ la fraction rationnelle

$$
f_{n}=\left[E\left(\alpha_{0}\right), \ldots, E\left(\alpha_{n}\right)\right]=E\left(\alpha_{0}\right)+\frac{1}{E\left(\alpha_{1}\right)+\frac{1}{\ddots+\frac{1}{E\left(\alpha_{n}\right)}}} .
$$

On définit également les polynômes $P_{n}$ et $Q_{n}$ pour $n \geq-2$, par récurrence et les égalités

$$
\begin{aligned}
& P_{-2}=0, \quad P_{-1}=1, \quad P_{n}=E\left(\alpha_{n}\right) P_{n-1}+P_{n-2}, \quad n \geq 0, \\
& Q_{-2}=1, \quad Q_{-1}=0, \quad Q_{n}=E\left(\alpha_{n}\right) Q_{n-1}+Q_{n-2}, \quad n \geq 0 .
\end{aligned}
$$

On a $f_{n}=P_{n} / Q_{n}$ et on dit que $P_{n}$ (resp. $Q_{n}$ ) est le numérateur (resp. le dénominateur $)$ de la nème réduite. On a aussi $\lim _{n \rightarrow \infty} \operatorname{deg}\left(\alpha-P_{n} / Q_{n}\right)=$ $-\infty$, ce qui justifie l'égalité $\alpha=\left[E\left(\alpha_{0}\right), E\left(\alpha_{1}\right), \ldots\right]$.

On peut maintenant définir la notion de $l$-normalité et de normalité :

DÉfinition 1. Un élément $\alpha \in \widetilde{K} \backslash k(x)$ est dit l-normal si ses quotients complets $\alpha_{n}$ sont de degré un pour tout $n \geq l$. Un élément $\alpha$ est dit normal si ses quotients complets sont tous de degré un sauf peut-être un nombre fini d'entre eux.

\section{Développement en fraction continue d'une fonction hyperel-} liptique. Soit $g$ un entier positif ou nul et soit $D$ un polynôme unitaire de degré $2 g+2$, à coefficients dans $k$ et sans racine multiple dans une clôture algébrique de $k$. On considère la série de Laurent à coefficients dans $k$, 


$$
y=x^{g+1}+\ldots \quad \text { vérifiant } y^{2}=D,
$$

puis le corps $K=k(x, y)$ quadratique sur $k(x)$. Une fonction quadratique est un élément de $K \backslash k(x)$ et comme $K$ est inclus dans $\widetilde{K}$, on peut parler du développement en fraction continue d'une fonction quadratique. Le calcul du développement en fraction continue d'une telle fonction est alors particulièrement simple; en effet on peut toujours la mettre sous la forme

$$
\alpha=\frac{L+f y}{M},
$$

où $L, M, f \in k[x], f \neq 0$ et $M$ divise $f^{2} D-L^{2}$. En effet soit $\alpha=(B+A y) / C$ avec $A, B, C \in k[x], A \neq 0, C \neq 0$, et soit $\lambda$ le quotient de $C$ par le p.g.c.d., noté $\delta$, de $C$ et de $A^{2} D-B^{2}$. Alors en posant $L=B \lambda, M=C \lambda, f=A \lambda$, on a $M \mid f^{2} D-L^{2}$. On peut trouver d'autres valeurs de $L, M, f$ mais le procédé décrit ci-dessus fournit le polynôme $f$ de plus petit degré si $A, B$ et $C$ sont premiers entre eux. On a alors [10]

$$
\alpha_{n}=\frac{L_{n}+f y}{M_{n}},
$$

où les polynômes $L_{n}$ et $M_{n}$ sont définis par les formules de récurrence suivantes : $L_{0}=L, M_{0}=M$ et pour $n \geq 1$,

$$
\begin{gathered}
L_{n}=E\left(\alpha_{n-1}\right) M_{n-1}-L_{n-1}, \\
M_{n} M_{n-1}=f^{2} D-L_{n}^{2} .
\end{gathered}
$$

On calcule $E\left(\alpha_{n-1}\right)$ par la formule

$$
E\left(\alpha_{n-1}\right)=E\left(\frac{L_{n-1}+B}{M_{n-1}}\right)
$$

où $B=E(f y)$.

On a besoin, dans cet article, de définir la notion de fonction quadratique "réduite". Un élément $\alpha \in K \backslash k(x)$ est dit réduit si $\operatorname{deg} \alpha \geq 1$ et $\operatorname{deg}\left(\alpha^{*}\right)$ $\leq-1$, où $\alpha^{*}$ désigne le conjugué de $\alpha$ dans $K$. C'est le cas pour $\alpha_{n}$, dans les paragraphes 3 et 4 , lorsque $n \geq 1$.

On a alors la proposition suivante :

Proposition 1. S'il existe $n_{0} \in \mathbb{N}$ tel que $\alpha_{n_{0}}$ soit réduit, alors $\alpha_{n}$ est réduit pour tout $n \geq n_{0}$ et on a

$$
\begin{gathered}
\operatorname{deg}\left(L_{n}+f y\right)=g+1+\operatorname{deg} f, \\
\operatorname{deg} M_{n} \leq g+\operatorname{deg} f .
\end{gathered}
$$

Preuve. Si $\alpha_{n_{0}}$ est réduit alors il est facile de voir que $\alpha_{n_{0}+1}$ l'est également. Dire que $\alpha_{n}$ est réduit est équivalent à dire que

$$
\operatorname{deg}\left(L_{n}-f y\right)<\operatorname{deg} M_{n}<\operatorname{deg}\left(L_{n}+f y\right) ;
$$

on en déduit que $\operatorname{deg} L_{n}=\operatorname{deg}(f y)=\operatorname{deg}\left(L_{n}+f y\right)$. 
COROllaire 1. Une fonction quadratique $\alpha$ telle que $\alpha_{1}$ soit réduite, est 1-normale si et seulement si pour tout $n \geq 1, \operatorname{deg} M_{n}=g+\operatorname{deg} f$.

Les notions de périodicité et de quasi-périodicité d'un développement en fraction continue d'une fonction de $K \backslash k(x)$ sont liées à celle de développement normal, comme on le verra par la suite. On rappelle celles-ci et quelques résultats les concernant.

DÉfinition 2. Un élément $\alpha \in K \backslash k(x)$ est dit périodique (resp. quasipériodique) s'il existe deux entiers $n \geq 0, \pi \geq 1$ tels que pour tout entier $m \geq n, \alpha_{m+\pi}=\alpha_{m}$ (resp. pour tout entier $m \geq n$, il existe un élément $c_{m} \in k^{*}$ tel que $\left.\alpha_{m+\pi}=c_{m} \alpha_{m}\right)$.

Si l'élément $\alpha$ est périodique (resp. quasi-périodique) le plus petit entier $\pi \geq 1$ vérifiant la propriété précédente est appelée la longueur de la période (resp. quasi-période).

On rappelle également les résultats suivants qui seront utilisés abondamment dans cet article et qui se trouvent dans [10].

Proposition 2. Soit $\alpha$ une fonction quadratique de la forme fy avec $f \in k[x] \backslash\{0\}$. Les propriétés suivantes sont équivalentes :

(i) L'élément fy est périodique.

(ii) L'élément fy est quasi-périodique.

(iii) Il existe $n \geq 1$ tel que $M_{n} \in k^{*}$ (où les polynômes $M_{n}$ sont définis par (2)).

(iv) Il existe $n \geq 1$ tel que $\operatorname{deg} \alpha_{n}=g+1+\operatorname{deg} f$.

(v) Le groupe des unités de l'anneau $\langle 1, f y\rangle=k[x, f y]$ n'est pas réduit $\grave{a} k^{*}$ (i.e. n'est pas trivial).

Proposition 3. Soit $\alpha \in K \backslash k(x)$. Les propriétés suivantes sont équivalentes:

(i) L'élément $\alpha$ est quasi-périodique.

(ii) Le groupe des unités $\mathcal{O}_{\alpha}^{*}$ de l'anneau des stabilisateurs $\mathcal{O}_{\alpha} d u k[x]$ module $\langle 1, \alpha\rangle:=k[x]+\alpha k[x]$ n'est pas réduit à $k^{*}$ (i.e. n'est pas trivial).

Enfin on sait que si $k$ est fini, un élément $\alpha \in K \backslash k(x)$ est toujours périodique, alors que si $k$ est infini (par exemple $k=\mathbb{Q}$ ), en général il ne l'est pas.

\section{Fractions continues et relations $A B C$}

Proposition 4. Soit $\alpha=f y / M, f, M \in k[x] \backslash\{0\}$, tel que $\operatorname{deg} M \leq$ $g+\operatorname{deg} f$ et $M$ divise $D$. Soient $P, Q \in k[x], P$ et $Q$ premiers entre eux. Alors la fraction rationnelle $P / Q$ ou $-P / Q$ est une réduite du développement en fraction continue de $\alpha$ si et seulement si il existe un polynôme $\widetilde{M} \in k[x]$, 
$\widetilde{M} \neq 0, \operatorname{deg} \widetilde{M} \leq g+\operatorname{deg} f$, tel que $(P, Q)$ soit solution non triviale (c'est$\grave{a}$-dire $P \neq 0$ et $Q \neq 0)$ de l'équation en $(U, V)$ :

$$
M^{2} U^{2}-f^{2} D V^{2}=\widetilde{M} M .
$$

Preuve. 1. Dans le sens direct, on écrit

$$
\alpha=\frac{P_{i-1} \alpha_{i}+P_{i-2}}{Q_{i-1} \alpha_{i}+Q_{i-2}}, \quad i \geq 0,
$$

et on remplace, dans cette égalité, $\alpha_{i}$ par sa valeur donnée en fonction des $L_{i}$ et $M_{i}$,

$$
\alpha_{i}=\frac{L_{i}+f y}{M_{i}},
$$

ce qui donne les relations

$$
\begin{aligned}
M_{i} M P_{i-2} & =f^{2} Q_{i-1} D-M P_{i-1} L_{i}, \\
M_{i} Q_{i-2} & =M P_{i-1}-Q_{i-1} L_{i} .
\end{aligned}
$$

On se sert alors de la relation

$$
\forall i \geq 0, \quad P_{i-1} Q_{i-2}-P_{i-2} Q_{i-1}=(-1)^{i},
$$

où l'on remplace $P_{i-2}$ et $Q_{i-2}$ en fonction de $Q_{i-1}, P_{i-1}, L_{i}, M_{i}$ à l'aide de (5); on trouve

$$
\forall i \geq 0, \quad M^{2} P_{i-1}^{2}-f^{2} D Q_{i-1}^{2}=(-1)^{i} M_{i} M .
$$

On a

$$
\alpha_{1}^{*}=\frac{1}{-f y / M-E(f y / M)},
$$

donc

$$
\operatorname{deg} \alpha_{1}^{*}=-\operatorname{deg} \frac{f y}{M}=\operatorname{deg} M-g-1-\operatorname{deg} f \leq-1,
$$

et donc $\alpha_{1}$ est réduit.

D'après la proposition 1 , on a,

$$
\forall i \geq 1, \quad \operatorname{deg} M_{i} \leq g+\operatorname{deg} f .
$$

2. Réciproquement, soit $(P, Q) \in(k[x])^{2}, P \neq 0, Q \neq 0, P$ et $Q$ premiers entre eux et vérifiant $\left(\mathcal{R}_{\widetilde{M}}\right)$. On écrit $\left(\mathcal{R}_{\widetilde{M}}\right)$ sous la forme

$$
(M P-f y Q)(M P+f y Q)=\widetilde{M} M .
$$

On suppose $\operatorname{deg}(f y Q)>\operatorname{deg}(M P)$; alors l'équation précédente donne

$$
2 \operatorname{deg}(f y Q) \leq g+1+\operatorname{deg} f+\operatorname{deg} \widetilde{M}
$$

et donc,

$$
g+1+\operatorname{deg} f \leq \operatorname{deg} \widetilde{M},
$$

ce qui contredit l'hypothèse $\operatorname{deg} \widetilde{M} \leq g+\operatorname{deg} f$. 
On suppose maintenant $\operatorname{deg}(f y Q)<\operatorname{deg}(M P)$. Alors on a

$$
2 \operatorname{deg}(f y Q)<2 \operatorname{deg} M P \leq g+\operatorname{deg} f+\operatorname{deg} \widetilde{M}
$$

et donc

$$
g+1+\operatorname{deg} f<\operatorname{deg} \widetilde{M},
$$

ce qui est encore impossible.

Finalement, on a $\operatorname{deg}(f y Q)=\operatorname{deg}(M P)$.

D'autre part on a les deux inégalités

$$
\left\{\begin{array}{l}
\operatorname{deg}(M P-f y Q) \leq \operatorname{deg}(f y Q), \\
\operatorname{deg}(M P+f y Q) \leq \operatorname{deg}(f y Q) .
\end{array}\right.
$$

Remarquons qu'elles ne peuvent pas être strictes toutes les deux et que l'on ne peut pas non plus avoir deux égalités; on a donc soit

$$
\left\{\begin{array}{l}
\operatorname{deg}(M P-f y Q)<\operatorname{deg}(f y Q) \\
\operatorname{deg}(M P+f y Q)=\operatorname{deg}(f y Q)
\end{array}\right.
$$

soit

$$
\left\{\begin{array}{l}
\operatorname{deg}(M P-f y Q)=\operatorname{deg}(f y Q), \\
\operatorname{deg}(M P+f y Q)<\operatorname{deg}(f y Q) .
\end{array}\right.
$$

Dans le premier cas l'équation $\mathcal{R}_{\widetilde{M}}$ donne

$$
\operatorname{deg}\left(\frac{P}{Q}-\frac{f y}{M}\right)=\operatorname{deg} \widetilde{M}-2 \operatorname{deg} Q-\operatorname{deg}(f y)
$$

et donc

$$
\operatorname{deg}\left(\frac{P}{Q}-\frac{f y}{M}\right) \leq-2 \operatorname{deg} Q-1<-2 \operatorname{deg} Q .
$$

Un résultat classique [8] permet de conclure que $P / Q$ est une réduite de $f y / M$. Dans l'autre cas, c'est $-P / Q$ qui est une réduite.

Corollaire 2. Soit $\alpha=f y / M, f, M \in k[x] \backslash\{0\}$, tel que $\operatorname{deg} M \leq$ $g+\operatorname{deg} f$. Alors $\alpha$ est 1 -normale ssi les équations $\left(\mathcal{R}_{\widetilde{M}}\right)$ sont impossibles en $(U, V)$ avec $U \neq 0, V \neq 0$ et $(U, V)=1$ et ceci pour tout $\widetilde{M} \in k[x] \backslash\{0\}$ avec $\operatorname{deg} \widetilde{M}<g+\operatorname{deg} f$.

Preuve. Remarquons que l'on a

$$
\forall i \geq 0, \quad M^{2} P_{i-1}^{2}-f^{2} D Q_{i-1}^{2}=(-1)^{i} M_{i} M
$$

et

$$
\forall i \geq 1, \quad P_{i-1} \neq 0, \quad Q_{i-1} \neq 0, \quad\left(P_{i-1}, Q_{i-1}\right)=1 .
$$

1. Supposons que $\alpha$ est 1-normale, qu'il existe $\widetilde{M} \in k[x] \backslash\{0\}$ avec $\operatorname{deg} \widetilde{M}<g+\operatorname{deg} f$ et que l'équation $\left(\mathcal{R}_{\widetilde{M}}\right)$ admette une solution non triviale :

$$
\exists(U, V) \in(k[x])^{2}, \quad U \neq 0, \quad V \neq 0, \quad(U, V)=1 .
$$


Alors la proposition 4 dit qu'il existe $i \geq 1$ et $\lambda \in k^{*}$ tels que

$$
U=\varepsilon \lambda P_{i-1}, \quad V=\lambda Q_{i-1}, \quad \text { avec } \varepsilon= \pm 1 .
$$

D'après $(6)$ et $\left(\mathcal{R}_{\widetilde{M}}\right)$ on a aussi

$$
\widetilde{M}=(-1)^{i} \lambda^{2} M_{i}
$$

D'autre part $\alpha_{i}=\left(L_{i}+f y\right) / M_{i}$ et comme $\alpha_{1}$ est réduit la proposition 1 donne,

$$
\operatorname{deg} \alpha_{i}=\operatorname{deg}\left(L_{i}+f y\right)-\operatorname{deg} M_{i}=g+1+\operatorname{deg} f-\operatorname{deg} \widetilde{M}>1,
$$

ce qui est absurde.

2. Réciproquement, on veut montrer que $\operatorname{deg} \alpha_{i}=1$ pour tout $i \geq 1$. On sait que $\alpha_{1}$ est réduit et donc pour tout $i \geq 1$,

$$
\operatorname{deg}\left(L_{i}+f y\right)=g+1+\operatorname{deg} f, \quad \operatorname{deg} M_{i} \leq g+\operatorname{deg} f .
$$

Supposons $\operatorname{deg} M_{i}<g+\operatorname{deg} f$. Alors la remarque faite au début de la preuve permet de dire que l'équation (6) a une solution non triviale, ce qui est impossible. Finalement on a $\operatorname{deg} M_{i}=g+\operatorname{deg} f$ et $\operatorname{deg} \alpha_{i}=1$.

Remarque. Si on appelle relation $A B C$ la donnée de trois polynômes $A, B, C \in k[x]$ tels que $A B C \notin k$, qui sont premiers entre eux deux à deux, et tels que

$$
A+B+C=0,
$$

on peut voir l'équation $\left(\mathcal{R}_{\widetilde{M}}\right)$ comme une relation $A B C$ où $A=M U^{2}, B=$ $-f^{2}(D / M) V^{2}, C=-\widetilde{M}$ à condition que $M U$ et $f D / M$ soient premiers entre eux.

On verra dans le paragraphe suivant que si cette condition n'est pas satisfaite on obtient toujours une relation $A B C$ qui provient de $\left(\mathcal{R}_{\widetilde{M}}\right)$.

Un outil essentiel pour la démonstration des résultats généraux est le théorème de Mason dans sa version caractéristique infinie [7] et finie [5]. Mais pour énoncer celui-ci on a besoin de la notion de radical (resp. radical modéré) d'un polynôme à coefficients dans $k$ de caractéristique infinie (resp. finie notée $p$ ).

DÉfinition. Soit $P \in k[x]$. On appelle radical (resp. radical modéré), et on note $S(P)$, le produit de tous les polynômes $x-\alpha$, où $\alpha$ est une racine de $P$ (resp. une racine de $P$ dont la multiplicité n'est pas divisible par $p$ ) dans une clôture algébrique de $k$.

ThÉorème De Mason. Soit une relation $A B C$ séparable (i.e. $A^{\prime} \neq 0$ ou $B^{\prime} \neq 0$ ) et soit $S$ le radical (resp. radical modéré) du produit $A B C$. Alors on $a$

$$
\max \{\operatorname{deg} A, \operatorname{deg} B, \operatorname{deg} C\}<\operatorname{deg} S
$$




\section{Résultats généraux}

ThÉORÈme 1. Soit $\alpha=f^{n} y / M, f, M \in k[x] \backslash\{0\}, f$ et $D$ premiers entre eux, $M \mid D, n$ un entier supérieur ou égal à 2 et $f$ unitaire, sans racine multiple dans une clôture algébrique de $k$. On suppose $\operatorname{deg} f \geq 1$ et $\operatorname{deg} M \leq n \operatorname{deg} f+g$.

(i) Si $k$ est de caractéristique infinie alors tous les quotients partiels du développement en fraction continue de $\alpha$ (sauf peut-être le premier) sont de degré inférieur ou égal à $1+2 g+\operatorname{deg} f$.

(ii) Soient $M_{i}$ les polynômes définis par (2). Si k est de caractéristique finie $p$ alors pour tout $i \geq 1$, on a

$$
\operatorname{deg} M_{i} \geq(n-1) \operatorname{deg} f-g
$$

et les quotients partiels sont de degré inférieur ou égal à $1+2 g+\operatorname{deg} f$, ou bien

$$
M_{i}=\widetilde{f}^{2}(x) \widetilde{D}(x) P\left(x^{p}\right),
$$

avec

$$
2 \operatorname{deg} \widetilde{f}+\operatorname{deg} \widetilde{D}+p \operatorname{deg} P<(n-1) \operatorname{deg} f-g
$$

où $\widetilde{f}\left|f^{n}, \widetilde{D}\right| D, P \in k[x] \backslash\{0\}$.

Preuve. (i) Caractéristique de $k$ infinie. En utilisant les notations introduites dans les paragraphes 2 et 3 où $n$ est remplacé par $i$ on a, parce que $\alpha_{1}$ est réduit puisque $\operatorname{deg} M \leq n \operatorname{deg} f+g$,

$$
\operatorname{deg} \alpha_{i}=\operatorname{deg}\left(L_{i}+f^{n} y\right)-\operatorname{deg} M_{i} \quad \text { si } i \geq 1 .
$$

D'après la proposition 1 , c'est $g+1+n \operatorname{deg} f-\operatorname{deg} M_{i}$. On veut montrer que

$$
g+1+n \operatorname{deg} f-\operatorname{deg} M_{i} \leq 1+2 g+\operatorname{deg} f,
$$

soit $\operatorname{deg} M_{i} \geq(n-1) \operatorname{deg} f-g$.

Montrons que les équations (6) sont impossibles si

$$
\operatorname{deg} M_{i}<(n-1) \operatorname{deg} f-g
$$

et ceci pour tout $i \geq 1$.

On pose $M_{i}=\widetilde{M}$ et on raisonne par l'absurde. On suppose qu'il existe une solution non triviale $U, V \in k[x] \backslash\{0\}$, telle que $U$ et $V$ soient premiers entre eux, à une équation

$$
\left(\mathcal{R}_{\widetilde{M}}\right) \quad M^{2} U^{2}-f^{2 n} D V^{2}=\widetilde{M} M,
$$

pour un certain polynôme $\widetilde{M} \neq 0$ vérifiant

$$
\operatorname{deg} \widetilde{M}<(n-1) \operatorname{deg} f-g .
$$

On va aboutir à une contradiction en utilisant le théorème de Mason. Celuici entraîne que si l'équation $\left(\mathcal{R}_{\widetilde{M}}\right)$ avec $A=M U^{2}, B=-f^{2 n}(D / M) V^{2}$, 
$C=-\widetilde{M}$ est séparable (i.e. $A^{\prime}$ ou $B^{\prime} \neq 0$ ) et si $A$ et $B$ sont premiers entre eux (ce qui est équivalent à dire que $M U$ et $f(D / M) V$ le sont) alors

$$
\max \left(\operatorname{deg} M U^{2}, \operatorname{deg} f^{2 n} \frac{D}{M} V^{2}, \operatorname{deg} \widetilde{M}\right)<\operatorname{deg}(U f D V \widetilde{M}),
$$

ce qui donne l'inégalité

$$
(n-1) \operatorname{deg} f-g-1<\operatorname{deg} \widetilde{M}
$$

et la contradiction.

Reste à voir que l'on obtient encore une relation $A B C$ qui permet d'avoir la contradiction si $(M U, f(D / M) V) \neq 1$. On a $(M, f)=1$ et $(M, D / M)=1$ car on a supposé $M \mid D,(D, f)=1$ et $D$ sans racine multiple. On pose $D=M D_{1},(M, V)=\delta_{1},\left(U, D_{1}\right)=\delta_{2}$, tous les p.g.c.d. étant supposés unitaires. On pose $M=\delta_{1} M_{1}, V=\delta_{1} V_{1}, U=\delta_{2} U_{1}, D_{1}=\delta_{2} D_{2}$. L'équation $\left(\mathcal{R}_{\widetilde{M}}\right)$ donne $M_{1} \delta_{1} \delta_{2}^{2} U_{1}^{2}-f^{2 n} \delta_{2} D_{2} \delta_{1}^{2} V_{1}^{2}=\widetilde{M}$, soit l'équation

$$
M_{1} \delta_{2} U_{1}^{2}-f^{2 n} D_{2} \delta_{1} V_{1}^{2}=\left(\delta_{1} \delta_{2}\right)^{-1} \widetilde{M}
$$

Montrons que l'on a $\left(M_{1} \delta_{2} U_{1}, D_{2} \delta_{1} V_{1}\right)=1$ :

$$
\left(M_{1}, D_{2}\right)=\left(M_{1}, \delta_{1}\right)=\left(\delta_{2}, D_{2}\right)=\left(\delta_{2}, \delta_{1}\right)=1,
$$

car $D$ est sans racine multiple, $\left(M_{1}, V_{1}\right)=\left(U_{1}, D_{2}\right)=1$ car $\delta_{1}=(M, V)$ et $\delta_{2}=\left(U, D_{1}\right)$, enfin $\left(\delta_{2}, V_{1}\right)=\left(U_{1}, \delta_{1}\right)=\left(U_{1}, V_{1}\right)=1$ car $(U, V)=1$. Cependant on peut avoir $\left(U_{1}, f^{n}\right) \neq 1$. Commençons par regarder le p.g.c.d. de $U_{1}$ et de $f$; posons $\left(U_{1}, f\right)=h_{1}, U_{1}=U_{2} h_{1}, f=f_{0} h_{1}, \widetilde{M}=\widetilde{M}_{1} \delta_{1} \delta_{2} h_{1}^{2}$. L'équation précédente donne l'équation

$$
M_{1} \delta_{2} U_{2}^{2}-f_{0}^{2 n} h_{1}^{2(n-1)} D_{2} \delta_{1} V_{1}^{2}=\widetilde{M}_{1} .
$$

On pose $A_{1}=M_{1} \delta_{2} U_{2}^{2}, B_{1}=-f_{0}^{2 n} h_{1}^{2(n-1)} D_{2} \delta_{1} V_{1}^{2}, C_{1}=-\widetilde{M}_{1}$.

On a $B_{1} \notin k^{*}$, car sinon $f_{0}$ et $h_{1}$ sont dans $k^{*}$; or par hypothèse $f$ n'est pas constant et $n \geq 2$. Supposons que les polynômes $A_{1}$ et $B_{1}$ soient premiers entre eux. Alors le théorème de Mason permet d'écrire

$$
\frac{1}{2}\left(\operatorname{deg}\left(M_{1} \delta_{2} U_{2}^{2}\right)+\operatorname{deg}\left(f_{0}^{2 n} h_{1}^{2(n-1)} D_{2} \delta_{1} V_{1}^{2}\right)\right)<\operatorname{deg}\left(M_{1} \delta_{2} U_{2} f_{0} h_{1} D_{2} \delta_{1} V_{1} \widetilde{M}_{1}\right) .
$$

En revenant aux données initiales, on obtient

$$
(n-1) \operatorname{deg} f-g-1+\operatorname{deg} \delta_{1}+\operatorname{deg} \delta_{2}+\operatorname{deg} h_{1}<\operatorname{deg} \widetilde{M},
$$

inégalité qui contredit (7).

Si les polynômes $A_{1}, B_{1}$ ne sont pas premiers entre eux, c'est que $U_{2}$ et $h_{1}$ ne le sont pas et que donc $h_{1} \notin k^{*}$, une étape de réduction supplémentaire est nécessaire. Raisonnons alors par récurrence et supposons qu'on soit arrivé à 
une équation $\left(\mathcal{R}_{\widetilde{M}_{i}}\right)$ avec $1 \leq i<n$,

$$
\left(\mathcal{R}_{\widetilde{M}_{i}}\right) \quad M_{1} \delta_{2} U_{i+1}^{2}-\left(\prod_{j=0}^{i-1} f_{j}^{2(n-j)}\right) h_{i}^{2(n-i)} D_{2} \delta_{1} V_{1}^{2}=\widetilde{M}_{i}
$$

avec $i \geq 1$, et où les $h_{j}, U_{j}, f_{j}, \widetilde{M}_{j}$ sont définis de la façon suivante :

$$
\begin{array}{lll}
\left(U_{j+1}, h_{j}\right)=h_{j+1}, & 0 \leq j \leq i-1 & \left(\text { on a posé } h_{0}=f\right), \\
U_{j+1}=U_{j+2} h_{j+1}, & 0 \leq j \leq i-1, & \\
h_{j}=f_{j} h_{j+1}, & 0 \leq j \leq i-1, & \\
\widetilde{M}_{i}=\widetilde{M}_{j+1} h_{j+1}^{2}, & 0 \leq j \leq i-1 \quad\left(\widetilde{M}=\widetilde{M}_{0} \delta_{1} \delta_{2}\right)
\end{array}
$$

on suppose de plus $h_{j} \notin k^{*}, 0 \leq j \leq i-1$.

Posons encore

$$
A_{i}=M_{1} \delta_{2} U_{i+1}^{2}, \quad B_{i}=\left(\prod_{j=1}^{i-1} f_{j}^{2(n-i)}\right) h_{i}^{2(n-i)} D_{2} \delta_{1} V_{1}^{2}, \quad C_{i}=-\widetilde{M}_{i}
$$

On a $B_{i} \notin k^{*}$ car sinon

$$
\left(\prod_{j=0}^{i-1} f_{j}^{2(n-j)}\right) h_{i}^{2(n-i)} \in k^{*}
$$

et donc $f_{0}, \ldots, f_{i-1} \in k^{*}$. Montrons alors que $h_{i} \notin k^{*}$. En effet, sinon comme on a $h_{i-1}=f_{i-1} h_{i}$ on aurait $h_{i-1} \in k^{*}$, ce qui contredit l'hypothèse de récurrence.

Finalement on a montré que $B_{i} \notin k^{*}$ car on a supposé $i<n$.

Supposons alors que $\left(U_{i+1}, h_{i}\right)=1$. Comme on a $B_{i} \notin k^{*}$ et $\left(A_{i}, B_{i}\right)=1$, le théorème de Mason entraîne que l'on a

$$
(n-1) \operatorname{deg} f-g-1+\operatorname{deg} \delta_{1}+\operatorname{deg} \delta_{2}+\sum_{j=1}^{i} \operatorname{deg} h_{j}<\operatorname{deg} \widetilde{M},
$$

inégalité qui contredit (7).

Supposons maintenant $U_{i+1}, h_{i}$ non premiers entre eux et posons

$$
\begin{aligned}
\left(U_{i+1}, h_{i}\right) & =h_{i+1} \quad\left(\notin k^{*} \text { et unitaire }\right), \\
U_{i+1} & =U_{i+2} h_{i+1}, \\
h_{i} & =f_{i} h_{i+1}, \\
\widetilde{M}_{i} & =\widetilde{M}_{i+1} h_{i+1}^{2} .
\end{aligned}
$$

L'équation $\left(\mathcal{R}_{\widetilde{M}_{i}}\right)$ donne

$\left(\mathcal{R}_{\widetilde{M}_{i+1}}\right) \quad M_{1} \delta_{2} U_{i+2}^{2}-\left(\prod_{j=0}^{i} f_{j}^{2(n-i)}\right) h_{i+1}^{2(n-i-1)} D_{2} \delta_{1} V_{1}^{2}=\widetilde{M}_{i+1}$

avec $h_{i+1} \notin k^{*}$. 
Reste à voir que, s'il ne s'est pas arrêté avant, au bout d'un nombre d'étapes égal à $n$ le processus s'arrête et permet de conclure. Supposons donc $i=n$. On a alors

$$
M_{1} \delta_{2} U_{n+1}^{2}-\left(\prod_{j=0}^{n-1} f_{j}^{2(n-j)}\right) D_{2} \delta_{1} V_{1}^{2}=\widetilde{M}_{n}
$$

On pose

$$
A_{n}=M_{1} \delta_{2} U_{n+1}^{2}, \quad B_{n}=-\left(\prod_{j=0}^{n-1} f_{j}^{2(n-j)}\right) D_{2} \delta_{1} V_{1}^{2}, \quad C_{n}=-\widetilde{M}_{n} .
$$

Montrons que $B_{n} \notin k^{*}$.

Supposons $B_{n} \in k^{*}$. Alors $\prod_{j=0}^{n-1} f_{j}^{2(n-j)} \in k^{*}$ et $\operatorname{donc} \operatorname{deg} f_{j}=0$ pour tout $0 \leq j \leq n-1$; mais alors $h_{j}=h_{j+1}, 0 \leq j \leq n-1$, donne $h_{0}=h_{1}=$ $\ldots=h_{n}=f$ et comme $\widetilde{M}=\delta_{1} \delta_{2} \prod_{j=1}^{n} h_{j}^{2} \widetilde{M}_{n}$ on aurait

$$
\operatorname{deg} \widetilde{M}=\operatorname{deg} \delta_{1}+\operatorname{deg} \delta_{2}+2 \sum_{j=1}^{n} \operatorname{deg} h_{j}+\operatorname{deg} \widetilde{M}_{n},
$$

donc $\operatorname{deg} \widetilde{M} \geq 2 n \operatorname{deg} f$, ce qui est absurde puisque nous avons supposé $\operatorname{deg} \widetilde{M}<(n-1) \operatorname{deg} f-g$.

(ii) Caractéristique de $k$ finie. On note par $p$ cette caractéristique. On doit montrer que si $\left(\mathcal{R}_{\widetilde{M}}\right)$ admet une solution non triviale $U, V \in k[x] \backslash\{0\}$, $U$ et $V$ premiers entre eux avec $0 \leq \operatorname{deg} \widetilde{M}<(n-1) \operatorname{deg} f-g$, alors on a $\widetilde{M}=\widetilde{f}^{2} \widetilde{D} P\left(x^{p}\right)$ avec $\widetilde{f}\left|f^{n}, \widetilde{D}\right| D, P \in k[x] \backslash\{0\}$, et $2 \operatorname{deg} \widetilde{f}+\operatorname{deg} \widetilde{D}+$ $p \operatorname{deg} P \leq(n-1) \operatorname{deg} f-g$.

Le théorème de Mason, tel qu'il est précisé dans [5] pour la caractéristique $p$, entraîne que la relation $A B C$ obtenue (après la réduction faite dans (i)) n'est pas séparable. On a donc, en utilisant les notations de (i),

$$
\widetilde{M}_{i}=P\left(x^{p}\right) .
$$

On a aussi

$$
\widetilde{M}=\delta_{1} \delta_{2} \prod_{j=1}^{i} h_{j}^{2} \widetilde{M}_{i} \quad \text { avec } \quad \delta_{1} \mid M \text { et } \delta_{2} \mid \frac{D}{M}
$$

et donc $\delta_{1} \delta_{2} \mid D$ car $D$ est supposé sans racine multiple.

Les polynômes $h_{j}$ étant par construction des diviseurs de $f$ et comme $i \leq n$, on a $\prod_{j=1}^{i} h_{j} \mid f^{n}$. Enfin la proposition 4 permet de dire que $\widetilde{M}$ est un certain polynôme $M_{j}$ pour un certain $j \geq 1$ (à une constante multiplicative près) tel qu'il a été défini par (2). 


\section{Application à l'étude de la quasi-périodicité}

5.1. Cas de la caractéristique infinie. On suppose que le corps $k$ est de caractéristique infinie. On se donne une fonction $\alpha \in K \backslash k(x)$. On considère l'anneau des stabilisateurs $\mathcal{O}_{\alpha}$ du $k[x]$-module $\langle 1, \alpha\rangle=k[x]+\alpha k[x]$. On sait qu'il existe un unique polynôme unitaire $f \in k[x]$ tel que

$$
\mathcal{O}_{\alpha}=\langle 1, f y\rangle \text {. }
$$

Considérons la factorisation de $f$ en polynômes irréductibles et unitaires sur $k$ :

$$
f=\prod_{i=1}^{r} f_{i}^{n_{i}},
$$

et supposons qu'il existe $i \geq 1$ tel que $\left(n_{i}-1\right) \operatorname{deg} f_{i}>g$ et $\left(f_{i}, D\right)=1$.

ThÉORÈme 2. Sous les hypothèses précédentes, le développement en fraction continue de $\alpha$ n'est pas quasi-périodique.

Preuve. 1. On va d'abord supposer que $\alpha=f^{n} y$, avec $(n-1) \operatorname{deg} f>g$, $f$ unitaire et sans racine multiple dans une clôture algébrique de $k,(f, D)=1$.

Dans ce cas, on sait (proposition 2) que le développement en fraction continue de $\alpha$ est quasi-périodique si et seulement si il existe un entier $i \geq 1$ tel que $M_{i} \in k^{*}$ (où les polynômes $M_{i}$ sont définis par (2)). Mais $M_{i} \in k^{*}$ équivaut à dire qu'il existe un entier $i \geq 1$ tel que $\operatorname{deg} \alpha_{i}=1+g+n \operatorname{deg} f$. Or le théorème 1 montre que $\operatorname{deg} \alpha_{i} \leq 1+2 g+\operatorname{deg} f$ pour tout $i \geq 1$. On a donc

$$
1+g+n \operatorname{deg} f \leq 1+2 g+\operatorname{deg} f
$$

soit $(n-1) \operatorname{deg} f \leq g$, ce qui contredit l'hypothèse $(n-1) \operatorname{deg} f>g$.

2. Cas général : $\alpha \in K \backslash k(x)$. D'une part, on se sert de la proposition 2 : " $\alpha$ est quasi-périodique si et seulement si le groupe des unités $\mathcal{O}_{\alpha}^{*}$ de l'anneau $\mathcal{O}_{\alpha}$ est non trivial".

D'autre part, on a les inclusions $\mathcal{O}_{\alpha}^{*} \subset \mathcal{O}_{f_{i}^{n_{i} y}}^{*}$ et les égalités $\mathcal{O}_{f_{i}^{n_{i} y}}^{*_{1}}=$ $\left\langle 1, f_{i}^{n_{i}} y\right\rangle$, $\operatorname{car}\left(f_{i}, D\right)=1$.

Considérons alors la fonction $f_{i}^{n_{i}} y$. Le polynôme $f_{i}$, étant irréductible sur $k$ de caractéristique infinie, est sans racine multiple dans une clôture algébrique de $k$, il est unitaire et on a par hypothèse $\left(n_{i}-1\right) \operatorname{deg} f_{i}>g$. On en déduit, grâce à la première partie de la démonstration, que le développement de $f_{i}^{n_{i}} y$ n'est pas quasi-périodique, ce qui revient à dire que $\mathcal{O}_{f_{i}^{n_{i}}{ }^{*}}^{*}=k^{*}$. Mais ceci entraîne que $\mathcal{O}_{\alpha}^{*}=k^{*}$.

5.2. Cas de la caractéristique finie. On suppose que la caractéristique de $k$ est finie et on considère $\alpha$ comme dans le théorème 1 .

DÉfinition. On appelle indice exceptionnel de $\alpha$ tout indice $i \geq 1$ tel que $\operatorname{deg} \alpha_{i}>1+2 g+\operatorname{deg} f$. 
ThÉORÈme 3. On suppose $p \gg 2 n \operatorname{deg} f+\operatorname{deg}(D / M)$. Soit $i$ un indice exceptionnel. Alors

$$
i \leq g+2 \quad \text { ou } \quad i>\frac{p-(2 n \operatorname{deg} f+\operatorname{deg}(D / M))}{1+g+n \operatorname{deg} f} .
$$

Preuve. 1. Soit $P_{j} / Q_{j}$ une réduite de $\alpha$, on a

$$
\forall j \geq 0, \quad j \leq \operatorname{deg} Q_{j} \leq(g+1+n \operatorname{deg} f) j .
$$

En effet, tous les $\alpha_{i}$ sont réduits pour $i \geq 1$ et ces inégalités résultent des inégalités établies dans la proposition 1 ,

$$
\forall j \geq 1, \quad 1 \leq \operatorname{deg} \alpha_{j} \leq g+1+n \operatorname{deg} f .
$$

2. On prend $V=Q_{i-1}$ dans $\left(\mathcal{R}_{\widetilde{M}}\right)$ et on a après réduction de $\left(\mathcal{R}_{\widetilde{M}}\right)$ une relation $A B C$, ce qui donne, avec les notations de la démonstration du théorème 1 ,

$$
\exists h\left|f^{2 n}, D_{2}\right| \frac{D}{M}, \delta_{1}=\left(M, Q_{i-1}\right), \text { tel que } \operatorname{deg}\left(h D_{2} \delta_{1} V_{1}^{2}\right) \equiv 0(\bmod p) .
$$

Comme $V=\delta_{1} V_{1}$ on en déduit que

$$
\operatorname{deg}\left(h D_{2} V^{2}\right) \equiv \operatorname{deg}\left(\delta_{1}\right)(\bmod p)
$$

et donc $\operatorname{deg} V^{2} \equiv \operatorname{deg} \delta_{1}-\operatorname{deg}\left(h D_{2}\right)(\bmod p)$. On pose $\beta=\operatorname{deg} \delta_{1}-\operatorname{deg}\left(h D_{2}\right)$. On a

$$
-2 n \operatorname{deg} f-\operatorname{deg} \frac{D}{M} \leq \beta \leq \operatorname{deg} \delta_{1} \leq \operatorname{deg} M \leq 2 g+2 .
$$

Montrons que $2 \operatorname{deg} Q_{i-1}=2 \operatorname{deg} V=\beta$ est impossible si $i>g+2$. En effet d'après $(8)$ et $(9)$, on aurait

$$
2(i-1) \leq \beta \leq 2 g+2,
$$

ce qui contredit $i>g+2$.

Par suite $2 \operatorname{deg} Q_{i-1}=\beta+\lambda p, \lambda \in \mathbb{N}^{*}$, et comme d'après (8) et (9) on a

$$
p-\left(2 n \operatorname{deg} f+\operatorname{deg} \frac{D}{M}\right) \leq 2 \operatorname{deg} Q_{i-1} \leq(g+1+n \operatorname{deg} f)(i-1)
$$

il vient

$$
i-1 \geq \frac{p-(2 n \operatorname{deg} f+\operatorname{deg}(D / M))}{1+g+n \operatorname{deg} f} .
$$

Corollaire 3. On se restreint ici aux fonctions $\alpha$ de la forme $f^{n} y$ avec $(n-1) \operatorname{deg} f>g, f$ unitaire sans racine multiple dans une clôture algébrique de $k$ et $(f, D)=1$. On suppose de plus $\alpha$ quasi-périodique et on note $\pi(\alpha)$ la longueur de sa quasi-période. Alors pour p assez grand, on a l'inégalité

$$
\pi(\alpha) \geq \frac{p-(2 n \operatorname{deg} f+\operatorname{deg} D)}{1+g+n \operatorname{deg} f} .
$$


Preuve. Comme on a supposé le développement de $\alpha$ quasi-périodique, on en déduit (proposition 2) l'existence d'une infinité d'indices $i$ tels que

$$
\operatorname{deg} \alpha_{i}=1+g+n \operatorname{deg} f .
$$

Mais comme on a aussi supposé $(n-1) \operatorname{deg} f>g$, on a, pour ces indices $i$,

$$
\operatorname{deg} \alpha_{i}>1+2 g+\operatorname{deg} f ;
$$

cette inégalité montre que ces indices sont exceptionnels. Le théorème 3 montre alors que pour $p$ assez grand le premier indice exceptionnel $i_{0}$ strictement supérieur à $g+2$ vérifie

$$
i_{0}>\frac{p-(2 n \operatorname{deg} f+\operatorname{deg} D)}{1+g+n \operatorname{deg} f} ;
$$

et donc pour $p$ assez grand,

$$
\pi(\alpha) \geq \frac{p-(2 n \operatorname{deg} f+\operatorname{deg} D)}{1+g+n \operatorname{deg} f}-g-2 .
$$

Reste à voir que pour tout $i$ tel que $1 \leq i \leq g+2$, on a $\operatorname{deg} \alpha_{i}<1+g+n \operatorname{deg} f$. En effet, sinon on a $\pi(\alpha) \leq g+2$, et donc avec l'inégalité (10), on obtient

$$
\frac{p-(2 n \operatorname{deg} f+\operatorname{deg} D)}{1+g+n \operatorname{deg} f}-g-2 \leq g+2 ;
$$

mais ceci est impossible pour $p$ est assez grand.

6. Le cas $k=\mathbb{Q}$ et sa réduction modulo $p$. On suppose maintenant $k=\mathbb{Q}$ et soit une fonction $\alpha$ dans $\mathbb{Q}(x, y) \backslash \mathbb{Q}(x)$ que l'on écrit sous la forme

$$
\alpha=\frac{L+f y}{M}
$$

avec $M \mid f^{2} D-L^{2}$ et $f$ unitaire.

Soit $p$ un nombre premier différent de 2. Alors on peut le choisir suffisamment grand pour que les conditions suivantes soient satisfaites :

(i) La réduction modulo $p$ du polynômes $D$, notée $\bar{D}$, existe et est sans racine multiple dans une clôture algébrique de $\mathbb{F}_{p}$.

(ii) Les réductions modulo $p$ des polynômes $L, f$ et $M$, notées $\bar{L}, \bar{f}, \bar{M}$ existent et vérifient $\bar{M} \neq 0,(\bar{f}, \bar{M})=1$.

On peut alors définir la fonction, notée $\bar{y}$, appartenant à $\mathbb{F}_{p}((1 / x))$ par $\bar{y}=x^{g+1}+\ldots$ et $\bar{y}^{2}=\bar{D}$. Considérons alors la fonction $\bar{\alpha}=(\bar{L}+\bar{f} \bar{y}) / \bar{M} \in$ $\mathbb{F}_{p}(x, \bar{y})$. Le corps des constantes étant fini, on sait que le développement en fraction continue de $\bar{\alpha}$ est quasi-périodique. On note $\pi_{p}(\bar{\alpha})$ la longueur de la quasi-période du développement en fraction continue de $\bar{\alpha}$.

L'étude de la suite $\left(\pi_{p}(\bar{\alpha})\right)$ passe par une analyse de l'anneau des stabilisateurs $\mathcal{O}_{\alpha} \mathrm{du} \mathbb{Q}[x]$-module $\langle 1, \alpha\rangle=\mathbb{Q}[x]+\alpha \mathbb{Q}[x]$, ainsi que des anneaux des 
stabilisateurs $\mathcal{O}_{\bar{\alpha}}$ des $\mathbb{F}_{p}[x]$-modules $\langle 1, \bar{\alpha}\rangle=\mathbb{F}_{p}[x]+\alpha \mathbb{F}_{p}[x]$. C'est l'objet du lemme qui suit :

Lemme 1. Si $(f, M)=1$, alors $\mathcal{O}_{\alpha}=\langle 1, f y\rangle$ et pour tout nombre premier $p$ assez grand, $\mathcal{O}_{\bar{\alpha}}=\langle 1, \bar{f} \bar{y}\rangle$.

Preuve. 1. L'anneau des stabilisateurs $\mathcal{O}_{\alpha}$ du module $\langle 1, \alpha\rangle$ est aussi celui du module

$$
\mathcal{M}=\langle M, L+f y\rangle .
$$

On vérifie que $f y \mathcal{M} \subset \mathcal{M}$ et donc

$$
\langle 1, f y\rangle \subset \mathcal{O}_{\alpha} .
$$

Pour montrer l'autre inclusion, on remarque que $\mathcal{O}_{\alpha}$ est inclus dans l'anneau des entiers de $\mathbb{Q}(x, y)$ et que celui-ci est $\mathbb{Q}[x, y]$, car $D$ n'a pas de racine multiple dans une clôture algébrique de $\mathbb{Q}$.

Par suite il s'écrit $\mathcal{O}_{\alpha}=\langle 1, h y\rangle, h \in \mathbb{Q}[x] \backslash\{0\}$. Mais alors $h$ doit vérifier

$$
h y M \in\langle M, L+f y\rangle
$$

et donc il existe $u, v \in \mathbb{Q}[x]$ tels que

$$
h y M=u M+v(L+f y),
$$

d'où $h M=v f$; mais comme $(M, f)=1$, on en déduit que $f \mid h$, ce qui montre l'autre inclusion.

2. Pour montrer que $\mathcal{O}_{\bar{\alpha}}=\langle 1, \bar{f} \bar{y}\rangle$, il suffit de remarquer que, pour $p$ assez grand, les polynômes $\bar{f}$ et $\bar{M}$ modulo $p$ restent premiers entre eux et que la démonstration précédente est indépendante du corps (on peut remplacer $\mathbb{Q}$ $\operatorname{par} \mathbb{F}_{p}$ ).

Considérons la factorisation de $f$ en polynômes irréductibles et unitaires $\operatorname{sur} \mathbb{Q}$ :

$$
f=\prod_{i=1}^{r} f_{i}^{n_{i}}
$$

et supposons qu'il existe $i$ tel que

$$
\left(n_{i}-1\right) \operatorname{deg} f_{i}>g \quad \text { et } \quad\left(f_{i}, D\right)=1 .
$$

ThÉORÈme 4. Sous les hypothèses précédentes, on a

$$
\lim _{p \rightarrow+\infty} \pi_{p}(\bar{\alpha})=+\infty .
$$

Preuve. 1. $\alpha=f^{n} y$ avec $(n-1) \operatorname{deg} f>g, f$ unitaire sans racine multiple dans une clôture algébrique de $\mathbb{Q}$ et $(f, D)=1$. On remarque que $\bar{\alpha}$ vérifie les hypothèses du corollaire 3 , en particulier le développement en fraction continue de $\bar{\alpha}$ est quasi-périodique, et donc d'après le corollaire 3 , pour $p$ 
assez grand, on a

$$
\pi_{p}(\bar{\alpha}) \geq \frac{p-(2 n \operatorname{deg} f+\operatorname{deg} D)}{1+g+n \operatorname{deg} f} .
$$

Soit $\varepsilon$ l'unité fondamentale de $\mathcal{O}_{\bar{\alpha}}^{*}$ de degré strictement positif. Montrons que $\operatorname{deg} \varepsilon \geq \pi_{p}(\bar{\alpha})$.

On a $\left\langle 1, \bar{\alpha}_{k}\right\rangle=\bar{\alpha}_{k} \ldots \bar{\alpha}_{1}\langle 1, \bar{\alpha}\rangle$ pour tout $k \geq 1$, et pour $k=\pi_{p}(\bar{\alpha})$,

$$
\left\langle 1, \bar{\alpha}_{\pi_{p}(\bar{\alpha})}\right\rangle=\langle 1, \bar{\alpha}\rangle
$$

On en déduit que $\varepsilon=\bar{\alpha}_{1} \ldots \bar{\alpha}_{\pi_{p}(\bar{\alpha})}$ est une unité de $\mathcal{O}_{\bar{\alpha}}=\langle 1, \bar{\alpha}\rangle$, et on sait que cette unité est l'unité fondamentale de degré strictement positif de $\mathbb{F}_{p}\left[x, \bar{f}^{n} \bar{y}\right]$. On en déduit aussi

$$
\operatorname{deg} \varepsilon=\sum_{i=1}^{\pi_{p}(\bar{\alpha})} \operatorname{deg} \bar{\alpha}_{i} \geq \pi_{p}(\bar{\alpha}),
$$

ce qui permet de conclure.

2. $\alpha \in \mathbb{Q}(x, y) \backslash \mathbb{Q}(x)$. On a $\mathcal{O}_{\alpha}=\langle 1, f y\rangle$ avec $f=\prod_{i=1}^{r} f_{i}^{n_{i}}$ et par hypothèse, il existe $i \geq 1$ tel que $\left(n_{i}-1\right) \operatorname{deg} f_{i}>g$. On sait que $\bar{\alpha}$ est quasi-périodique puisque $\mathbb{F}_{p}$ est fini et que cette quasi-périodicité est liée à l'existence d'une unité non triviale de l'anneau des stabilisateurs du $\mathbb{F}_{p}[x]$ module $\langle 1, \bar{\alpha}\rangle=\mathbb{F}_{p}[x]+\bar{\alpha} \mathbb{F}_{p}[x]$ (proposition 3).

Plus précisément si on note $\bar{\alpha}_{i}$ les quotients complets du développement en fraction continue de $\bar{\alpha}$, on a pour tout $k>l \geq 1$,

$$
\left\langle 1, \bar{\alpha}_{k}\right\rangle=\bar{\alpha}_{k} \ldots \bar{\alpha}_{l}\left\langle 1, \bar{\alpha}_{l-1}\right\rangle .
$$

Soit alors $l \geq 1$ tel que $\bar{\alpha}_{l-1}$ est réduit et soit $k=\pi_{p}(\bar{\alpha})+l-1$; on a

$$
\left\langle 1, \bar{\alpha}_{\pi_{p}(\bar{\alpha})+l-1}\right\rangle=\left\langle 1, \bar{\alpha}_{l-1}\right\rangle .
$$

Ceci montre que $\bar{\alpha}_{\pi_{p}(\bar{\alpha})+l-1} \ldots \bar{\alpha}_{l}=\varepsilon$ est une unité de $\mathcal{O}_{\bar{\alpha}}$, c'est même l'unité fondamentale de degré positif. On a remarqué (lemme 1) que $\mathcal{O}_{\bar{\alpha}}=\langle 1, \bar{f} \bar{y}\rangle$, il existe donc $i$ tel que

$$
\mathcal{O}_{\bar{\alpha}} \subset \mathcal{O}_{\bar{f}_{i}^{n_{i}} \bar{y}} \quad \text { avec } \quad\left(n_{i}-1\right) \operatorname{deg} \bar{f}_{i}>g .
$$

Comme $f_{i}$ est irréductible sur $\mathbb{Q}$, il n'a pas de racine multiple dans une clôture algébrique de $\mathbb{Q}$ et par suite pour $p$ assez grand $\bar{f}_{i}$ possède la même propriété. Soit $\eta$ l'unité fondamentale de $\mathcal{O}_{\bar{f}_{i} n_{i}}$ telle que $\operatorname{deg} \eta>0$. Comme $\mathcal{O}_{\bar{\alpha}}^{*}$ est un sous-groupe de $\mathcal{O}_{\bar{f}^{n}{ }_{i} \bar{y}}^{*}$ il existe un entier $t \geq 1$ et $\lambda \in \mathbb{F}_{p}^{*}$ tels que

$$
\varepsilon=\lambda \eta^{t} \text {. }
$$

Mais alors

$$
\operatorname{deg} \varepsilon=t \operatorname{deg} \eta
$$


En utilisant en outre la première partie de la démonstration où $\alpha$ vaut $f_{i}^{n_{i}} y$, on a les inégalités suivantes :

$$
\frac{p-\left(2 n_{i} \operatorname{deg} f_{i}+\operatorname{deg} D\right)}{1+g+n_{i} \operatorname{deg} f_{i}} \leq \pi_{p}\left(\bar{f}_{i}^{n_{i}} \bar{y}\right) \leq \operatorname{deg} \eta \leq \operatorname{deg} \varepsilon
$$

et

$$
\operatorname{deg} \varepsilon=\sum_{i=l}^{\pi_{p}(\bar{\alpha})+l-1} \operatorname{deg} \bar{\alpha}_{i} \leq(g+1+n \operatorname{deg} f) \pi_{p}(\bar{\alpha})
$$

et donc $\lim _{p \rightarrow+\infty} \pi_{p}(\bar{\alpha})=+\infty$.

Les théorèmes 2 et 4 suggèrent la conjecture suivante :

Conjecture. Soit $\alpha \in \mathbb{Q}(x, y) \backslash \mathbb{Q}(x)$. On suppose que l'anneau des stabilisateurs du module $\langle 1, \alpha\rangle$ est l'anneau $\langle 1, f y\rangle$. Alors la réduction modulo un nombre premier $p \neq 2$ existe pour tout $p$ suffisamment grand et on a

$$
\lim _{p \rightarrow+\infty} \pi_{p}(\bar{\alpha})=+\infty \Leftrightarrow \text { fy non quasi-périodique. }
$$

7. Etude des fonctions $x^{n} y$ et $x^{n} / y$ dans le cas particulier où $g=0$ et la caractéristique du corps $k$ est infinie. On pose ici $D=x^{2}+u x+v$.

Proposition 5. On suppose que la caractéristique de $k$ est infinie et on se donne un entier $n \geq 2$.

(i) Si $v \neq 0$ alors tous les quotients partiels de $x^{n} y$ et $x^{n} / y$ sont tous de degré 1 ou 2 sauf peut-être le premier.

(ii) Si $v=0$ alors $x^{n} y$ et $x^{n} / y$ sont 1 -normales.

Preuve. Le (i) est un corollaire du théorème 1, le (ii) n'est pas un corollaire du théorème 1 mais un corollaire de la démonstration, ce qui veut dire que si on reprend cette preuve on montre, grâce au théorème de Mason, que les équations

$$
M^{2} U^{2}-x^{2 n} D V^{2}=\widetilde{M} M
$$

sont impossibles en $(U, V) \in(k[x])^{2}$, $U$ et $V$ premiers entre eux et ceci pour tout $\widetilde{M}$ avec $0 \leq \operatorname{deg} \widetilde{M}<n$, où $M=1$ ou $D$.

Ce dernier résultat peut être vu comme un prolongement du travail de D. G. Cantor [4].

Enfin pour illustrer ces résultats théoriques, on termine par deux exemples :

EXEMPLE 1. Le développement en fraction continue de $x^{2} y$ où $k=\mathbb{Q}$ ou $\mathbb{F}_{p}, p \geq 3$ et où $D=x^{2}+2$. Avec les notations du deuxième paragraphe 
et en posant $L_{0}=0, M_{0}=1, f=x$, on a pour tout $n$ supérieur ou égal à 1 ,

$$
\left\{\begin{array}{l}
L_{2 n-1}=x^{3}+\frac{x}{n} \\
M_{2 n-1}=-\frac{x^{2}}{n^{2}} \\
E\left(\alpha_{2 n-1}\right)=-2 n^{2} x \\
L_{2 n}=x^{3}-\frac{x}{n} \\
M_{2 n}=-2 n(n+1) x^{2}+1 \\
E\left(\alpha_{2 n}\right)=-\frac{x}{n(n+1)}
\end{array}\right.
$$

On en déduit le développement de $x^{2} y$ avec $k=\mathbb{Q}$ :

$$
x^{2} y=\left[x^{3}+x,-2 x,-\frac{x}{2},-8 x,-\frac{x}{6}, \ldots,-2 n^{2} x,-\frac{x}{n(n+1)}, \ldots\right],
$$

puis le développement avec $k=\mathbb{F}_{p}$ :

$$
\overline{x^{2} y}=\left[x^{3}+x, \overline{-2 x, \frac{p-1}{2} x, \ldots,-2 x, 2 x^{3}+2 x}\right]
$$

et donc $\pi_{p}\left(\overline{x^{2} y}\right)=2(p-1)$.

EXEMPLE 2. Le développement en fraction continue de $x^{2} / y$ où $k=\mathbb{Q}$ ou $\mathbb{F}_{p}, p \geq 3$ et où $D=x^{2}+2$. On pose $L_{0}=0, M_{0}=D, f=x$. Pour tout $n$ supérieur ou égal à 1 on a

$$
\left\{\begin{array}{l}
L_{2 n-1}=x^{3}+\frac{2 x}{2 n-1} \\
M_{2 n-1}=-\frac{2 x^{2}}{(2 n-1)^{2}} \\
E\left(\alpha_{2 n-1}\right)=-(2 n-1)^{2} x \\
L_{2 n}=x^{3}-\frac{2 x}{2 n-1} \\
M_{2 n}=-(2 n+1)(2 n-1) x^{2}+2 \\
E\left(\alpha_{2 n}\right)=-\frac{2 x}{(2 n+1)(2 n-1)}
\end{array}\right.
$$

et donc si $k=\mathbb{Q}$ :

$$
\frac{x^{2}}{y}=\left[x,-x,-\frac{2}{3} x,-9 x, \ldots,-(2 n-1)^{2} x,-\frac{2 x}{(2 n+1)(2 n-1)}, \ldots\right]
$$

et si $k=\mathbb{F}_{p}$ :

$$
\left(\overline{\frac{x^{2}}{y}}\right)=\left[x, \overline{-x, \ldots,-4 x, x^{3}+x,-4 x, \ldots,-x}\right], \quad \pi_{p}\left(\overline{\frac{x^{2}}{y}}\right)=p-1 .
$$


Cette fonction quadratique est quasi-périodique et périodique, sa longueur de période est $2(p-1)$. On remarque que $\bar{\alpha}_{1}=2 \bar{\alpha}_{p}$.

\section{Références}

[1] J.-P. Allouche, A. Lubiw, M. Mendès France, A. J. van der Poorten and J. Shallit, Convergents of folded continued fractions, Acta Arith. 77 (1996), 77-96.

[2] J.-P. Allouche, M. Mendès France and A. J. van der Poorten, An infinite product with bounded partial quotients, ibid. 59 (1991), 171-182.

[3] L. Baum and M. M. Sweet, Continued fractions of algebraic power series in characteristic 2, Ann. of Math. 103 (1976), 593-610.

[4] D. G. Cantor, On the continued fractions of quadratic surds, Acta Arith. 68 (1994), 295-305.

[5] Y. Hellegouarch, Analogues en caractéristique $p$ d'un théorème de Mason, C. R. Acad. Sci. Paris Sér. I 325 (1997), 141-144.

[6] D. E. Knuth, The Art of Computer Programming, Vol. 2, Seminumerical Algorithms, 2nd printing, 1969, pp. 360ff.

[7] R. C. Mason, Diophantine Equations over Function Fields, Cambridge Univ. Press, 1984 .

[8] B. de Mathan, Approximations diophantiennes dans un corps local, Bull. Soc. Math. France Suppl. Mém. 21 (1970), 1-93.

[9] M. Mendès France and A. J. van der Poorten, Some explicit continued fraction expansions, Mathematika 38 (1991), 1-9.

[10] R. Paysant-Le Roux, Périodicité des fractions continues dans un corps de fonctions hyperelliptiques, Arch. Math. (Basel) 61 (1993), 46-58.

Département de Mathématiques

Université de Caen

Campus II - B.P. 5186

14032 Caen Cedex, France

E-mail: hellegou@math.unicaen.fr paysant@math.unicaen.fr 